\title{
PRINCIPLE OF ALTERNATING GRADIENT ACCELERATION
}

\author{
Ming Xie, Lawrence Berkeley National Laboratory, Berkeley, CA94720, USA
}

\begin{abstract}
Principle of alternating gradient acceleration is proposed to overcome one of the major limitations on laser acceleration: phase slippage due to the difference between phase velocity of acceleration wave and longitudinal speed of accelerated particle. According to the principle, net acceleration can be achieved in various ways even under continuous phase slippage. Single particle dynamics of both longitudinal and transverse motions are investigated under alternating gradient acceleration.
\end{abstract}

\section{INTRODUCTION}

In all known accelerators, acceleration gradient is kept positive along the entire passage of accelerated particles. This is so for a good reason, of course, as synchronous acceleration or all-positive-gradient acceleration can be achieved so easily that there is no need to do it otherwise. However, situation can be drastically different when acceleration wavelength is scaled down, where phase slippage becomes a critical problem limiting net acceleration.

For direct field laser acceleration, major difficulties have been encountered when trying to maintain synchronous acceleration. In all schemes proposed so far, the approach to overcome phase slippage is to either place structures in the near field of intense laser and particle beams or load gases directly in the passage of the beams, in an effort to either terminate the interaction or slow down the acceleration wave. As a result, hefty price has to be paid by sacrificing acceleration gradient and beam current, and in doing so the very attractiveness of the laser acceleration schemes is severely compromised. Phase slippage is also a major limitation for ponderomotive driven acceleration in all laserplasma-based schemes.

In this report, Principle of Alternating Gradient Acceleration (PAGA) is proposed to overcome the phase slippage problem. According to the principle, net acceleration can be achieved in various ways even under continuous phase slippage. Several schemes based on the principle have been proposed to achieve high average gradient for both direct field and ponderomotive driven accelerations $[1,2,3,4,5]$. Therefore, synchronous acceleration, a concept so deeply rooted in the conventional accelerator physics and practice, is neither a necessary condition nor worthy of striving for in order to achieve high gradient laser acceleration. It is now the time to cross over a major conceptual threshold.

The dramatic change of operation principle from synchronous acceleration to alternating gradient acceleration may have profound impact on the beam dynamics we used to know. The main purpose of this report, therefore, is to establish a new foundation for both longitudinal and trans- verse dynamics, and reveal some unique characteristics of beam dynamics under the new principle.

Taking a perspective in a broader scale, PAGA is proposed as one of the two pillars for a new landscape of laser accelerations. The other pillar, Concept of Oversized Open Waveguide (COOW), is required to overcome other two major limitations on laser acceleration: diffraction of laser field and structure damage by high power laser. By supporting each other in harmony, the two pillars together support a great variety of architectures that are capable of overcoming simultaneously the three major limitations and hence to provide high gradient acceleration over much extended length in single stage with durable solid-state acceleration structures. Readers are referred to my recent articles $[1,2,3,4,5,6,7]$ for a more complete exposition of the entire framework.

\section{THE PRINCIPLE}

Longitudinal field of a traveling wave seen by a charged particle moving on an orbit defined by $t(z)$ is of the form

$$
\mathcal{E}[z, t(z)]=E(z) \cos \psi(z) .
$$

Assuming phase velocity of the wave and longitudinal speed of the particle are different, the particle is accelerated over a distance $L_{a}$ corresponding to a $\pi$ phase slippage, and then decelerated over $L_{d}$ correspondingly to another $\pi$ phase slippage. Respectively, the energy gain and loss are

$$
\begin{gathered}
\Delta W_{a}=\int_{0}^{L_{a}} E(z) \cos \psi(z) d z=q E_{a} L_{a} T_{a}, \\
\Delta W_{d}=\int_{L_{a}}^{L_{2 \pi}} E(z) \cos \psi(z) d z=-q E_{d} L_{d} T_{d},
\end{gathered}
$$

where $L_{2 \pi}=L_{a}+L_{d}, E_{a}\left(E_{d}\right)$ is the magnitude of peak acceleration (deceleration) field, and $T_{a}\left(T_{d}\right)$ is a transit factor over the distance $L_{a}\left(L_{d}\right)$ satisfying $0<T_{a} \leq 1(0<$ $\left.T_{d} \leq 1\right)$. The average gradient over $L_{2 \pi}$ is then

$$
G_{2 \pi}=\frac{\Delta W_{a}+\Delta W_{d}}{L_{a}+L_{d}}=G_{a} \frac{\left[1-\left(\frac{E_{d}}{E_{a}}\right)\left(\frac{L_{d}}{L_{a}}\right)\left(\frac{T_{d}}{T_{a}}\right)\right]}{1+\frac{L_{d}}{L_{a}}},
$$

where $G_{a}=\Delta W_{a} / L_{a}>0$ is the average gradient over $L_{a}$. Thus, the condition requiring net energy gain over a $2 \pi$ phase slippage follows

$$
G_{2 \pi}>0 \quad \Longrightarrow\left(\frac{E_{d}}{E_{a}}\right)\left(\frac{L_{d}}{L_{a}}\right)\left(\frac{T_{d}}{T_{a}}\right)<1
$$

Equation (2) is a general statement of the principle of alternating gradient acceleration, applicable to all types of accelerations under continuous phase slippage. It also reveals the variety of approaches in achieving net acceleration. 


\section{LONGITUDINAL DYNAMICS}

We now discuss single particle dynamics of longitudinal motion in the field of Eq.(1) under continuous phase slippage. In particular, we consider direct field acceleration by an electromagnetic wave confined in a waveguide. For electron with $q=-e$, energy equation can be written as

$$
\frac{d \gamma}{d z}=-k a \cos \psi
$$

where

$$
a=\frac{e E(z) \lambda}{2 \pi m c^{2}}, \quad \psi=\omega t-\int_{0}^{z} k_{z}(s) d s .
$$

The electron phase may be separated into two parts, $\psi=$ $\psi_{l}+\psi_{\gamma}$, where $\psi_{l}$ depends on accelerator lattice

$$
\psi_{l}=\int_{0}^{z} d s\left[k-k_{z}(s)\right],
$$

and $\psi_{\gamma}$ depends on particle's energy and initial phase

$$
\psi_{\gamma}=\int_{0}^{z} d s k\left(\frac{1}{\beta(s)}-1\right)+\psi_{0}, \quad \psi_{0}=\omega t_{0} .
$$

We are interested in the relativistic regime satisfying

$$
\left|\frac{d \psi_{l}}{d z}\right| \gg\left|\frac{d \psi_{\gamma}}{d z}\right| .
$$

Consequently, we may define a lattice period, $L_{2 \pi}$, according to the condition for a $2 \pi$ phase slippage by

$$
\left|\Delta \psi_{l}\left(\Delta z=L_{2 \pi}\right)\right|=2 \pi .
$$

In addition, we introduce $a(z)=a_{s}(z) f_{l}(z)$, where $a_{s}(z)$ is assumed to vary slowly in the scale $L_{2 \pi}, f_{l}(z)$ and $k_{z}(z)$ are periodic functions with period $L_{2 \pi}$ and $0<f_{l}(z) \leq 1$.

Taking average of Eq.(3) over the fast scale $L_{2 \pi}$, one gets

$$
\frac{d \bar{\gamma}}{d z}=-k a_{s}\left(C_{l} \cos \psi_{\gamma}-S_{l} \sin \psi_{\gamma}\right),
$$

where $\bar{\gamma}=<\gamma>, C_{l}=<f_{l} \cos \psi_{l}>, S_{l}=<f_{l} \sin \psi_{l}>$ are averaged quantities. Obviously, net energy exchange is possible if $C_{l} \neq 0$ or $S_{l} \neq 0$. For convenience we may set the lattice such that $C_{l}=0$ and $S_{l}>0$. Hence, longitudinal equations of motion in slow scale become

$$
\frac{d \bar{\gamma}}{d z}=k a_{s} S_{l} \sin \psi_{\gamma}, \quad \frac{d \psi_{\gamma}}{d z}=\frac{k}{2 \bar{\gamma}^{2}} .
$$

It is important to note that Eq.(4) is casted into the same form as the usual equations of motion for a linac [8]. As a result, our entire wealth of knowledge about synchronous acceleration can be directly applied to the case of alternating gradient acceleration. For example, period of small amplitude synchrotron oscillation follows directly from Eq.(4)

$$
\lambda_{s}=\frac{\bar{\gamma}^{3 / 2} \lambda}{\sqrt{a_{s} S_{l} \cos \psi_{\gamma}}} .
$$

\section{TRANSVERSE DYNAMICS}

Transverse motion of an electron under direct field alternating gradient acceleration by a $T M$ mode can be derived from the radial equation of motion

$$
\frac{d\left(\gamma r^{\prime}\right)}{d z}=-\frac{e}{m c^{2}}\left(E_{r}-\beta c \mu_{0} H_{\phi}\right),
$$

where $r^{\prime}=d r / d z$. The transverse field components can be related to the acceleration field, $E_{z}(r, z, t)=$ $J_{0}\left(k_{\perp} r\right) \mathcal{E}(z, t)$, in paraxial approximation by [8]

$$
\begin{gathered}
E_{r}(r, z, t)=\left.r \frac{\partial E_{r}}{\partial r}\right|_{r=0}=-\frac{r}{2 \epsilon} \frac{\partial(\epsilon \mathcal{E})}{\partial z}, \\
H_{\phi}(r, z, t)=\left.r \frac{\partial H_{\phi}}{\partial r}\right|_{r=0}=\frac{\epsilon r}{2} \frac{\partial \mathcal{E}}{\partial t},
\end{gathered}
$$

where $\epsilon=\epsilon_{0} \epsilon_{1}, \epsilon_{1}=\nu_{1}^{2}, \nu_{1}=1+\delta \nu_{1}$. Hence from Eq.(6)

$$
\frac{d\left(\gamma r^{\prime}\right)}{d z}=\frac{e r}{2 m c^{2}}\left[\frac{\partial \mathcal{E}}{\partial z}+\frac{\beta \epsilon_{1}}{c} \frac{\partial \mathcal{E}}{\partial t}+\left(\frac{1}{\epsilon_{1}} \frac{\partial \epsilon_{1}}{\partial z}\right) \mathcal{E}\right] .
$$

Upon introducing reduced variable $Q=\sqrt{\gamma} r$ [9], Eq.(7) can be converted into the form of Hill's equation

$$
Q^{\prime \prime}+K_{r} Q=0
$$

where

$$
\begin{gathered}
K_{r}=\frac{k^{2}}{\gamma}\left(\frac{a^{2} \cos ^{2} \psi}{4 \gamma}-\frac{a \sin \psi}{2 \gamma^{2}}+a \delta \nu_{1} \sin \psi-a b \cos \psi\right), \\
b=\frac{\lambda}{2 \pi} \frac{\partial \delta \nu_{1}}{\partial z} .
\end{gathered}
$$

There are four small parameters: $1 / \gamma \ll 1, a \ll 1,|b| \ll$ 1 and $\left|\delta \nu_{1}\right| \ll 1$. To compare the relative magnitude of $b$ and $\delta \nu_{1}$, it is convenient to introduce a profile function

$$
f_{d}(z)=\frac{1}{1+e^{-\left(z+L_{d} / 2\right) / \Delta}}-\frac{1}{1+e^{-\left(z-L_{d} / 2\right) / \Delta}},
$$

where $L_{d}$ is the width of the profile, $\Delta$ is the width of rising or falling edge, and $0 \leq f_{d} \leq 1$. Note, with $\Delta / L_{d} \ll 1$, $f_{d}$ approaches a square profile. Using Eq.(9), we have

$$
\left|\frac{b}{\delta \nu_{1}}\right|_{\max }=\frac{\lambda}{2 \pi}\left|\frac{\partial \ln \left(f_{d}\right)}{\partial z}\right|_{\max }=\frac{\lambda}{2 \pi \Delta} .
$$

Thus, for highly relativistic particle in a medium loaded structure with slow rising and falling edges satisfying $(\lambda / 2 \pi \Delta) \ll 1, K_{r}$ is dominated by one term

$$
K_{r}=\frac{k^{2} a \delta \nu_{1} \sin \psi}{\gamma} .
$$

To realize alternating gradient acceleration, we consider a specific scheme $[1,2]$ in which an oversized open waveguide supporting $T M_{01}$ mode is periodically loaded along $z$ direction with plasma layers, each of width $L_{d}$ and separated by a vacuum of distance $L_{a}$. The plasma density profile can be specified by $n(z)=n_{0} f_{d}(z)$, corresponding to 
a reduction in the index of refraction $\delta \nu_{1}=-f_{d}(z) / 2 \gamma_{p}^{2}$, where $\gamma_{p}=\omega / \omega_{p} \gg 1$ and $\omega_{p}$ is the plasma frequency associated with the peak density $n_{0}$. In this scheme, $E_{a}=$ $E_{d}=E, T_{a}=T_{d}=2 / \pi, f_{l}=1$, and

$$
S_{l}=\frac{2}{\pi}\left(\frac{1-\xi}{1+\xi}\right)
$$

where $\xi=L_{d} / L_{a}$, which varies in the range $\{0-1\}$, and under the condition $\Delta / L_{d} \ll 1, f_{d}$ is approximated by a square profile, shifted in $z$ from Eq.(9)

$$
f_{d}(z)=\left\{\begin{array}{lll}
0 & : & 0 \leq z \leq L_{a} \\
1 & : & L_{a}<z \leq L_{2 \pi}
\end{array}\right.
$$

When considering dynamics in the fast scale, we may neglect slow synchrotron motion by setting $\psi_{\gamma}=\psi_{0}$, and furthermore, $\psi_{0}=\pi / 2$ for maximum acceleration, thus

$$
\begin{gathered}
\frac{d \gamma}{d z}=k a \sin \psi_{l}, \\
K_{r}(z)=-\kappa f_{d}(z) \cos \psi_{l}(z),
\end{gathered}
$$

where $\kappa=k^{2} a / 2 \gamma \gamma_{p}^{2}$. Noting that $k_{z}=k \nu_{1}\left(1-1 / 2 \gamma_{g}^{2}\right)$ $[1,2,4,5]$, where $\gamma_{g}$ is a quantity determined by the waveguide, the lattice phase $\psi_{l}$ can be expressed as

$$
\psi_{l}(z)=\left(\frac{k}{2 \gamma_{g}^{2}}\right) z+\left(\frac{k}{2 \gamma_{p}^{2}}\right) \int_{0}^{z} f_{d}(s) d s .
$$

Thus by definition, we obtain from Eq.(13), using Eq.(10)

$$
L_{a}=\gamma_{g}^{2} \lambda, \quad L_{d}=\frac{\gamma_{p}^{2} \lambda}{1+\left(\gamma_{p} / \gamma_{g}\right)^{2}} .
$$

Finally, the lattice phase $\psi_{l}$ can be reduced to

$$
\psi_{l}(z)= \begin{cases}\frac{\pi}{L_{a}} z & : \quad 0 \leq z \leq L_{a} \\ \frac{\pi}{L_{d}}\left(z-L_{a}\right)+\pi & : \quad L_{a} \leq z \leq L_{2 \pi} .\end{cases}
$$

Indeed, with Eq.(14), we observe $C_{l}=0$.

Given Eqs. $(8,10,12,14)$, an alternating gradient focusing lattice is fully specified. Applying the smooth approximation [10] to the lattice, the $\beta$-function is found to be

$$
\beta_{t}=\left(\frac{\gamma m c^{2}}{G_{2 \pi}}\right) f_{\xi}
$$

where

$$
\begin{gathered}
G_{2 \pi}=\frac{d\left(\bar{\gamma} m c^{2}\right)}{d z}=e E S_{l}, \\
f_{\xi}=\frac{4}{\pi \sqrt{2 \xi(1+\xi)-16 \xi^{2} / \pi^{2}}} .
\end{gathered}
$$

It is noted that because of the scaling $\beta_{t} \sim \gamma$, electron beam size remains constant during acceleration for the AG focusing lattice discussed here. Thus, the acceptable value for normalized rms beam emittance can be determined by the condition

$$
\epsilon_{n} \leq \frac{G_{2 \pi} \sigma_{\max }^{2}}{m c^{2} f_{\xi}},
$$

where $\sigma_{\max }$ is the maximum rms beam size limited by considerations of waveguide aperture and beam dynamics.

\section{EXAMPLE}

An example of alternating gradient acceleration is given in table 1 for highly relativistic electron satisfying the conditions $\left(\gamma / \gamma_{g}\right)^{2} \gg 1$ and $\left(\gamma / \gamma_{p}\right)^{2} \gg 1$. The example is taken from [2], although the acceleration scheme was also presented in [1]. In this example, direct field acceleration by $T M_{01}$ mode is taken place in a capillary waveguide periodically loaded with plasma layers. Taking $\sigma_{\max } / R=$ 0.1 , it then follows from Eq.(16) that $\epsilon_{n} \leq 0.25 \mathrm{~mm}$-mrad. For electron energy of $1 \mathrm{GeV}$, Eq.(5) gives $\lambda_{s}=4.9 \mathrm{~m}$ assuming $\cos \psi_{\gamma}=1$ for maximum longitudinal focusing, and Eq.(15) gives $\beta_{t}=3.1 \mathrm{~m}$.

Table 1. Direct Field Alternating Gradient Acceleration

\begin{tabular}{|c|c|c|c|c|c|}
\hline$\lambda[\mu \mathrm{m}]$ & 1 & $P_{0}[\mathrm{TW}]$ & 10 & $E_{a}[\mathrm{GV} / \mathrm{m}]$ & 1.9 \\
\hline$R / \lambda$ & 200 & $n_{0}\left[10^{17} / \mathrm{cm}^{3}\right]$ & 1.1 & $E_{s}[\mathrm{GV} / \mathrm{m}]$ & 1.5 \\
\hline$\nu_{2}$ & 1.5 & $L_{a t t n}[\mathrm{~m}]$ & 5.3 & $G_{2 \pi}[\mathrm{GeV} / \mathrm{m}]$ & 1 \\
\hline$\gamma_{g}$ & 328 & $L_{a}[\mathrm{~cm}]$ & 10.8 & $\Delta W_{a}[\mathrm{MeV}]$ & 127 \\
\hline$\gamma_{p}$ & 100 & $L_{d}[\mathrm{~cm}]$ & 0.91 & $\Delta W_{d}[\mathrm{MeV}]$ & -11 \\
\hline$S_{l}$ & 0.53 & $f_{\xi}$ & 3.1 & $a_{s}\left[10^{-3}\right]$ & 0.59 \\
\hline
\end{tabular}

\section{CONCLUSIONS}

The analysis of single particle dynamics reveals no showstopper for alternating gradient acceleration. In fact, the longitudinal dynamics in the slow scale is shown to be identical to that for synchronous acceleration. On transverse dynamics, the AG focusing inherent from the direct field acceleration is found to be surprisingly favorable for its weaker focusing strength, resulting in much relaxed alignment tolerance and smaller emittance growth. Although the dynamics presented here is focused on direct field acceleration, the general dynamical characteristics under alternating gradient acceleration are expected to hold for ponderomotive driven acceleration as well. Effects of plasma wakefields driven by laser and particle beams will be treated elsewhere. This work was supported by the U.S. Department of Energy under contract No.DE-AC03-76SF00098.

\section{REFERENCES}

[1] M. Xie, PAC99 Conf. Proc., 3678 (1999).

[2] M. Xie, LBNL-42783, 1999.

[3] M. Xie, LBNL-42784, 1999.

[4] M. Xie, AIP Conf. Proc. 472 for AAC Workshop, 701 (1998).

[5] M. Xie, EPAC98 Conf. Proc., 830 (1998).

[6] M. Xie, LBNL-40558, 1997.

[7] M. Xie, PAC97 Conf. Proc., 660 (1997).

[8] R. Helm and R. Miller, in Linear Accelerators, edited by P. Lapostolle and A. Septier (North-Holland, 1969), p115.

[9] M. Reiser, Theory and Design of Charged Particle Beams, (Wiley, 1994), p77.

[10] K. Symon et al., Physical Review, 103, 1837 (1956). 